\title{
Application of Robotics in Dentistry
}

Manjusha Rawtiya ${ }^{1}, K_{\text {Kvita Verma }}{ }^{2}$, Priyank Sethi ${ }^{3}$, Kapil Loomba ${ }^{4}$

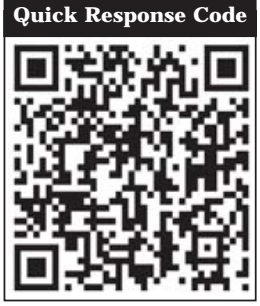

doi: $10.5866 / 2014.641696$

1,2\&3Senior Lecturer

Conservative Dentistry \& Endodontics

Peoples Colege Of Dental Sciences, Bhopal, India

${ }^{4}$ Prof \& Head

Department Of Conservative Dentistry \& Endodontics, Career Postgraduate Institute Of Dental Sciences,

Bhopal, India

\section{Article Info:}

Received: J uly 9, 2014

Review Completed: August 10, 2014

Accepted: November 8, 2014

Available Online: J anuary, 2015 (www.nacd.in)

(C) NAD, 2015 - All rights reserved

\section{Email for correspondence:}

manju27mona@gmail.com

\section{Introduction}

Robotics is the branch of technology that deals with the design, construction, operation, and application of robots as well as computer systems for their control, sensory feedback, and information processing. The term robotics was introduced by writer Isaac Asimov in his science fiction book, I Robot, published in 1950. ${ }^{1}$

The term "robot" was coined by the Czech Playright Karel Capek in 1921 in his play Rossom's Universal Robots. ${ }^{2}$ It is from the check word 'robota' which means forced labor. According to the Robot Institute of America, a robot is defined as "a reprogrammable, multifunctional manipulator designed to move material, parts, tools, or specialized devices through various programmed motions for the performance of a variety of tasks" (1979). ${ }^{3}$ It is a machine capable of carrying out a complex series of action automatically. Robotics is not yet used in dentistry even though all the

\section{ABSTRACT:}

The success of applying robotics to the medical field has opened a new frontier with vast areas for expansion and exploration; more specifically, robotics with dental application is a relatively untraveled area to pursue. It was introduced in the field of medicine and dentistry to increase precision, quality and safety of various procedures. The main aim of this paper was to review current research in the field of dental robotics. A review of literature was performed using el ectronic searching methods of Pub Med database and Google for the applications of robots in Dentistry. Specific keywords were used and theelectronic search yiel ded around 87 articles out of which 30 articles were included based on predetermined criteria in the fields of dentistry. By pooling the extracted data from selected papers, the reviewed data was synthesized.

Key words: Endo Micro Robots, Nanorobots, Simroid, Robotic dental Drill, Showa Hanako, Geminoid DK

necessary technol ogies havealready been devel oped and could easily be adapted. ${ }^{4}$

\section{Robotics in Medical Field}

Following the developments in industrial robot technology, robotics has found its way into the medical field and is used in a range of surgical disciplines. It has been decades since robots have been in the field of medicine and this technology would not had been true if Leonardo da Vinci would not have created many robot-like sketches and designs in the 1500. It all started way back in 1985, when a robot, the PUMA 560 was used to place a needle for a brain biopsy using CT guidance. ${ }^{5}$ In 1988, the MROBOT, was used to perform prostatic surgery by Dr. Senthil Nathan at Guy's and St Thomas' Hospital, London. Further, the first complete robotic surgery took place at The Ohio StateU niversity Medical Center under the direction of Dr. Robert E. Michler, Professor and Chief, Cardiothoracic Surgery. ${ }^{6}$ Advancements poured in

\section{Indian Journal of Dental AdVAnCEMEnTS Journal homepage: www. nacd. in}


J uly 1998 when a reconnection of the fallopian tubes operation was performed successfully in Cleveland using ZEUS. ${ }^{7}$ On May 12, 2008, the first imageguided MR-compatible robotic neurosurgical procedure was performed at University of Calgary by Dr. Garnette Sutherland using the NeuroArm. ${ }^{8}$ Also, revolution came in when in J anuary 2009, the first all-robotic-assisted kidney transplant was performed at Saint Barnabas Medical Center in Livingston, New J ersey by Dr. Stuart Geffner. ${ }^{9}$ In September 2010, the first robotic operation at the femoral vasculature was performed at the University Medical Centre Ljubljana by a team led by Borut Geršak. The robot used was the first true robot, meaning it was not simply mirroring the movement of human hands, but was guided by pressing on buttons. ${ }^{10}$

\section{Robots in Dental Field}

\section{A) Dental patient robot}

Dental therapy skills often depend on the competence and ability of clinicians and it is necessary for them to have extensive experience using methods and models that accurately reflect actual treatment procedures and conditions. Recently fresh pass out graduates lack clinical skills and experience in treating patients. Until recently, clinical training was carried out on consenting volunteer patients. However, recent changes in ethical issues related to environmental studies, medicine and dentistry have made such clinical training difficult. Currently so called 'Phantoms' consist of simple functional cephalic region and arrangement of teeth which is different from actual patients. As a result concept of dental patient robot was initiated in J apan (Figure 1).

\section{Showa Hanako}

Tokyo's Showa University engaged robotics company Tmsuk to manufacture the realistic robot which is designed to simulate a number of typical patient gestures and responses, allowing dental students to experience what it's like to work with a real patient. Showa Hanako 2 is said to be a more user friendly and functional replacement to Showa Hanako 1, which was presented in March 2010. "Love doll" maker Orient I ndustry is responsible for the silicone skin (this replaces the PVC skin of the previous model) and mouth lining, which increases the realistic feel and prevents water from getting into the machinery. It can blink, roll its eyes, sneeze, shake its head, cough, move its tongue and even get tired when having to keep its mouth open for too long. Interestingly, the robot is also capable of simulating a gag reflex, which is quite frequent during dental procedures. J apanese engineers also used a speech recognition technology developed by Raytron to facilitate conversation capability. ${ }^{11}$

\section{Geminoid DK}

Hiroshi Ishiguro,(Professor at Osaka University) together with his colleagues at J apan's Advanced Telecommunications Research Institute International, created a new robot called Geminoid DK.( Figure -3) The most interesting fact about the invention is that it represents the accurate replica of Aalborg University Professor Henrik Scharfe. It is worth mentioning that the robot is the first in a series based on personalities outside Japan. According to the inventor, the machine is intended to push forward android science and philosophy, in searching for answers to fundamental questions. The Geminoids can be remotely controlled, being equipped with advanced motion-capture technology. The latter allows the machine to mimic facial expressions and precisely imitate head motions. ${ }^{12}$

\section{Simroid}

It is a super realistic dental training robot for dentists devel oped at The Nippon Dental University Kokoro with dental equipment maker Morita Manufacturing. It is actually an upgrade to Simuloid, a less sophisticated dental training robot created back in 2007.I ts creators claim that Simroid, a next-generation dental patient simulator, has been developed to provide more emotional feedback to dentists in training. What set it apart is advancements in robotics and artificial intelligence that now make it react with more lifelike and emotional responses. Sensors in and around the mouth allow it to feel simulated pain and discomfort, which it will react to negatively, making students more conscious of their technique. It can even react with discomfort when the dentist-to-be's elbow comes into contact with its breast, so its creators have thought of everything. A new artificial skin has been used instead of silicone, which can easily tear when the robot has to open its mouth wide, and Simroid is now been equipped with far better communication skills. Speech recognition capabilities allow it to respond and react to questions or commands. It's even able to rate and evaluate their treatment, with two cameras monitoring the student's every move, and readings from its sensors being recorded throughout the procedure. ${ }^{13}$

This robot patient is manufactured by Kokoro, who also make the Actroid line of lifelike humanoid robots. It has been developed to improve student patient communication skills by emphasizing attitude rather than technique. ${ }^{14}$ 


\section{B) Endo Micro Robot}

Success of endodontic treatment depends on clinician knowledge, expertise including his or her tactile senseand judgment. Endodontic mishaps like perforation, canal ledging, apical foramen transportation and stripping, excessive instrumentation beyond apex, inadequate or improper canal preparation and instrument separation may occur during root canal preparation. ${ }^{15}$ To reduce the potential for human error and improve the quality of endodontic treatment, it is necessary to develop advanced endodontic technology innovation by applying advanced engineering and computer aided technology. ${ }^{16,} 17$ The Advanced Endodontic Technol ogy Devel opment project consists of four subsubjects:

(1) Development of a technique to thoroughly assess thetooth's condition using 2-dimensional $\mathrm{x}$-ray images to build a computer 3-D tooth model, displaying state-of the-art computer graphics;

(2) Development of an automatic prescription system from the 3-D root canal model, using computer-aided treatment procedure planning;

(3) Design and build a smart multi-purpose precision micro machine to perform automated root canal treatment;

(4) Develop a new ultrasonic cleaning tool with pressure assisted jetting/vacuum waste removal.

This computer-controlled machine will be mounted on several teeth within the patient's mouth. With on-line monitoring and intelligent control, the micro machine or robot will perform the automated drilling, cleaning, and filling of the root canal. All other sub-project results will be incorporated into this robotic operation.

Specific objectives for micro robot design include:

(1) Reducing the reliance on the skills of the dentist,

(2) Minimizing human error, and

(3) Offering a method for precise diagnosis and treatment. ${ }^{18}$

\section{Features of Micro Endo Robot}

- A micro-position and orientation adjustment to ensure that the tools start at a precise point;

- An automatic feed rateand travel distancecontrol to ensure that the tools can reach the required canal depth and stop at a designated point;

- Built-in micro sensors to monitor the probing and drilling/reaming process;
- Apex sensing and control to prevent root perforations or the potential to over shoot (exceeding the apex of the canal);

- Flexible drills or files to allow for cleaning and shaping curved canals.

- Vacuum attachments capable of sucking the debris or loose tissue from the root canal and/or pressurized solution jets to flush the chips away.

- A preliminary quantitative study established the design requirements.

In order to provide accurate positioning of the tool, with correct angular orientation, an ideal basic machine must have five degrees of freedom to control the following axes:

- $\quad \mathrm{X}$-axis, along the teeth row, with travel range of $5 \mathrm{~mm}$;

- $\quad \mathrm{Y}$-axis, across the teeth row, with travel range of $4 \mathrm{~mm}$;

- Z-axis, the tool advancement direction, perpendicular to the tooth occlusal surface, with a travel range of $15 \mathrm{~mm}$ minimum. When using a longer tool, the endodontic tool should be able to reach $28 \mathrm{~mm}$ from the tooth crown, covering the required range of treatment;

- The angular adjustment of the tool entrance angle of $\pm 12^{\circ}$ in the X-Z plane;

- The angular adjustment of the tool entrance angle of \pm 12 in the $Y-Z$ plane. The size of the machine must be compact enough to fit into the patient's mouth and sit on the teeth between his/her two jaws. The dimension should be within $20 \mathrm{~mm}$ x $20 \mathrm{~mm}$ x $28 \mathrm{~mm}$;

- The spindle must have the rotational power to drive the tool at speeds and torque used in endodontic treatment tools;

- The machine should be able to provide a thrust force not less than 500gm for tool penetration into the crown and dentin.

The machine has a saddle-shaped base. It will ride on a pair of reference brackets and the teeth. Before taking X-rays and mounting this machine, the brackets, in assorted sizes to fit the patients' teeth must be pre-clamped firmly on the tooth to be treated. Neighboring teeth may be used as support. The bracket pair provides three radiopaque reference points for the machine registration, thus establishing a coordination system. Once the machine is seated on the reference bracket, the machine base will have no motion relative to the patient's teeth, regardless of the patient's head or jaw movements. The machine is designed to be compact and rigid so that the patient can bite on it. 


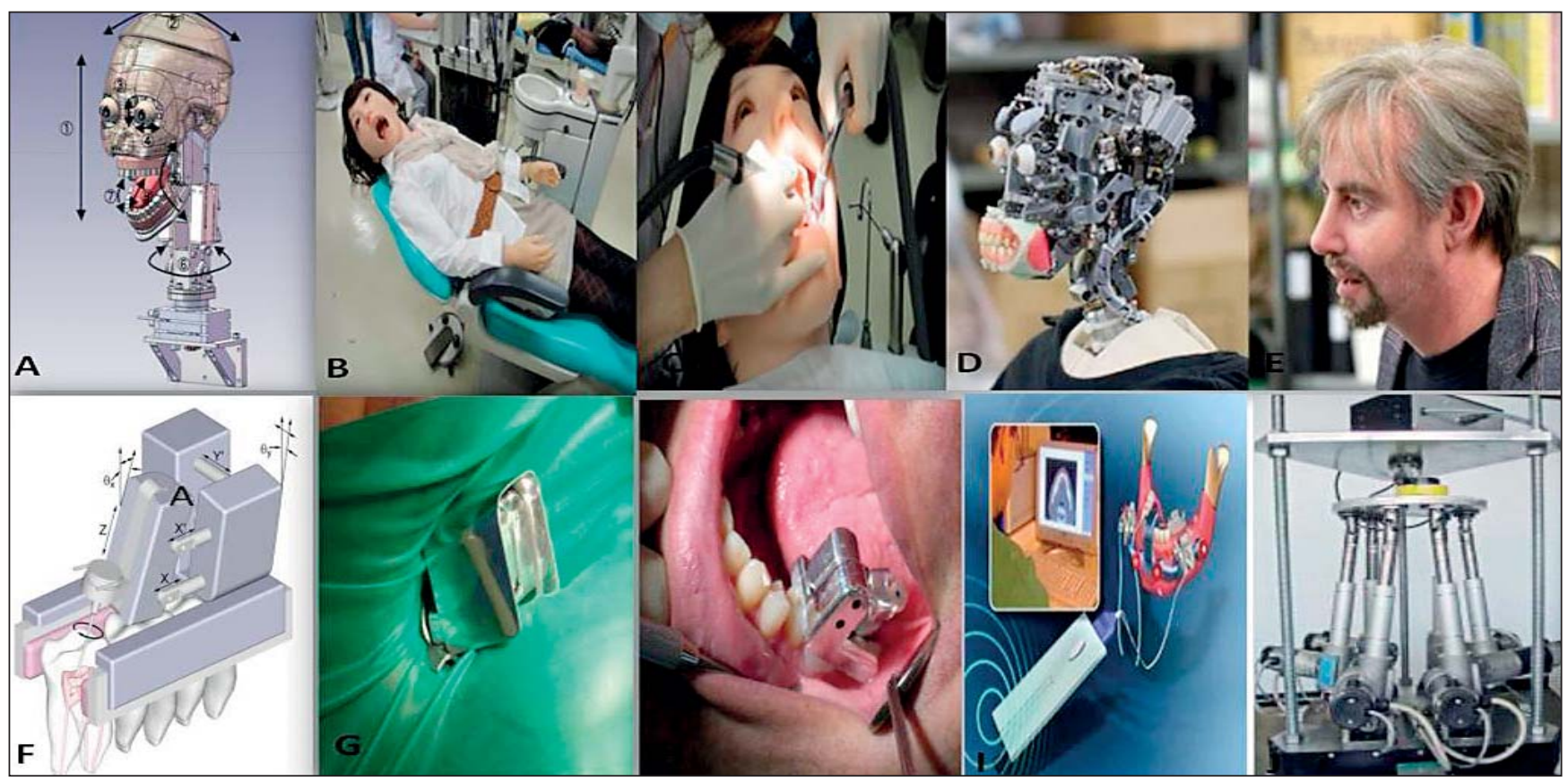

Figure 1: Concept of dental patient robot initiated in J apan.

There is no need for the patient to actively keep his/ her mouth wide open, nor is there a need for the patient to keep his/her head absolutely still. Designed for multi-purpose use, this machine can hold various endodontic tools and auxiliary devices. With a quick tool change approach, utilizing a cartridge design, different tools can be pre-mounted on a small modular unit, which can be inserted into a sliding adaptor on the $Z$ axis.

\section{Micro Sensors, Actuators, and Control Systems}

This machine design also incorporates sensors for intelligent monitoring of the treatment process. Because the compact features of the sensors, they can be fabricated using a surface micro machine method to producesilicon-on-insulator (SOI) wafers, which will beembedded in the micro robot. Six micro actuators are used to control the five axes (five degrees of freedom) and the on/off spindle of the tool. Each actuator is independently controlled by a digital NC controller. The controller should react the sensor signal quickly, typically in a few milliseconds.

Auxiliary functions provided include: an irrigation nozzle for cleaning, a vacuum suction cup for chip and waste fluid removal, and/or optical fibers for lighting, imaging and observation. A manual remote control will be provided for the clinician, but a fully automatic operation with computer-aided treatment procedure planning and control is the ultimate goal for a zero-defect operation. An interface system will be provided for the clinician to interact with the machine control.

The computer-aided treatment process planning system, with functions similar to CAD/CAM programs in the machinery industry, will generate the standard NC codes (G codes and M codes) as output from a computer aided design file. These codes will control the movements of the robot through an NC digital controller. This computer prescription program is being developed to automatically select the appropriate tool and to determine:

(1) Thetool's starting point, position, and direction,

(2) The tool's path;

(3) The tool's stopping point,

(4) The cutting parameters, such as speed and feed, and

(5) The geometry of the 3-D tooth model after treatment. The computer program will plan the sequence of the operations using a variety of tools and motion control parameters to complete the preparation of the root canal. An optimization program will be integrated into this auto prescription program to minimize the removal of tooth structure and to eliminate unnecessary tool change.

The development of an endodontic micro robot is the center piece of Advanced Endodontic Technol ogy Devel opment. Compared to conventional 
access methods for root canal treatment, this research presents a less invasive method using automated access and canal preparation during endodontic therapy thus reducing human errors.

\section{C) Dental Nanorobots}

Nanorobotics is the technology of creating machines or robots at or close to the microscopic scale of 2 nanometers. Nanorobots represent microscopical objects artificially capable of free diffusion inside the human body and which can interact with human body cells or can manipulate them, with a nanometric resolution (10-9 m) in order to fulfill their tasks. As a regular robot, a nanorobot can be manufactured out of thousands of mechanical parts, made out of nanomaterials such as carbon nanotubes, metallic nanoconductors and diamondoid materials. ${ }^{19}$

Multiple Nanorobots working on the teeth in unison, invisible to the naked eye, may be used for cavity preparation and restoration of teeth. The cavity preparation is very precisely restricted to the demineralized enamel and dentin, thus providing maximum conservation of sound tooth structure. ${ }^{20}$ A colloidal suspension containing millions of active analgesic micron-size dental robots will be instilled on the patient's gingival to reach the dental pulp where once installed will shut down all sensitivity in any particular tooth that requires treatment. After oral procedures are completed, the dentist orders the Nanorobots to restore all sensation, to relinquish control of nerve traffic and to egress from the tooth by similar pathways used for ingress. ${ }^{21}$ Reconstructive dental Nanorobots could selectively and precisely occlude specific tubules within minutes, offering patients a quick and permanent cure. ${ }^{22}$

\section{D) Surgical Robots}

In the last decade, surgery and robotics have reached a maturity that has allowed them to be safely assimilated to create a new kind of operating room in medical field. This new environment includes robots for local surgery and telesurgery, audiovisual telecommunication for telemedicineand teleconsultation, robotic systems with integrated imaging for computer-enhanced surgery, and virtual reality $(V R)$ simulators enhanced with haptic feedback, for surgical training. ${ }^{23}$ According to Satava, "the operating room of the future will be a sophisticated mix of stereo imaging systems, micro robots, robotic manipulators, virtual reality/ tel epresence workstations, and computer integrated surgery." 24
A surgical robot system for maxillofacial surgery has been developed. With this system the surgeon interactively programs the robot during the surgery after which the robot performs the pre-programmed tasks. ${ }^{25}$

\section{Sensor-Equipped I mplant Setup}

Dental I mplant is a surgical treatment of tooth root replacement which is the most frequently used in prosthetic dentistry. Present mechanical guided system or template drill guided system is an oldfashion technology for dental implant guidance. It is derived by waxing up patient's teeth impression, and then it has to transfer the interactive planning of implants from 2D and 3D visualization to the template in reality. ${ }^{26}$ The drawbacks of this method are on the lack of interactive control during implant is operated, as well as, the high cost of drilled guided template intervention. ${ }^{27,28}$ Currently, a new system of computer-assisted surgery (CAS) for oral implantology application has been developed. It includes preoperative and intraoperative procedures. The preoperative surgery is to use 3D views as provided to enhance raw images obtained from the patient before operation. The technique is to render a target region and a pathway associated with relative organs from CT data. This helps dentists to get familiar with the anatomy of the patient in advance. ${ }^{29}$ Turning to intraoperative stage, intraoperative support can beused during the real surgical procedure in both navigation and decision aid purposes. It provides three dimensional (3D) orientation of surgical instrument position and trajectory displayed on a monitor in real-time within patient's 3D imaging data. ${ }^{30} \mathrm{~A}$ robust and high accuracy tracking system is main parameters in navigation system.

Sets of infrared (IR) based surgical marker emitters are designed in different patterns for tracking movement of surgical instruments and patient's position. Because the objects except the surgical markers in the operation room become invisible, it is the main benefit of the infrared based marker.

\section{Rosy}

It is a new computer- aided intra-operative guidance system for implant surgery .For implant planning and surgery with the robot system Rosy; five different work processes are required. Preprosthetic planning consist of preparation of direct mock up with the help of radio- opaque resin on set of pre - operative cast. This mock up is tried on the patient mouth. The cast with mock up is placed on lower support plate of Rosy and a pointer is set in 
the upper support plate that marks the planned implant entry site and angulation. Using the six step motors, the lower support plate can be adjusted in all the six degrees of spatial freedom. Implant position can be determined by aiming the planned entry point of the implant on the plaster surface. Subsequently the template is placed on the cast and orientation of the implant adjusted if necessary so that the abutment construction will be located within the crown contour. Finally the pointer is exchanged by drill and required holes are driven into template. Prefabricated titanium tubes are clamped into theses hole, so that their direction is clearly visible in subsequent radiograph. Implant position is corrected with the help of Osirix software. These tubes are removed from the template, the holes closed with a clear cold curing resin and the template is reinforced in the cervical area. Afterwards the cast with the template is placed on Rosy and the motors move automatically to the position saved during the initial implant planning. The correction values from Osirix are now entered into Rosy. The cast is thus shifted and rotated according to the correction values and the corrected position is obtained in relation to the originally planned position. The template is now placed in the mouth of the patient. The drill handles can be inserted directly into the holes in the template; the guidance is so accurate that the usage of metal sleeves is not necessary. ${ }^{31}$

\section{ROBOTIC DENTAL DRILL}

Developed by Tactile Technologies, based in Rehovot, Israel is designed to take the complexity out of dental implant work. It could make the procedure cheaper, quicker and less painful for patients. Tactile Technologies has already tested parts of the system on animals and recently received approval from the US Food and Drug Administration to begin trials on humans.

Procedure consists of clamping a frame onto a patient's jaw and very thin needles penetrate the gum to determinethe location of the bone. This data is wirelessly transmitted to a PC, which combines it with CT scan data to configurea set of drill guides. The guides are then attached to the frame and finally the dentist presses a button to start the drilling in the precise location required. Once activated the drill is self-guiding but the practitioner can still alter the drilling process at any time. The system causes fewer traumas to the patient. ${ }^{32}$

\section{Dental Robot Cerec}

The joint venture between i Robot, theinventor of the home robot vacuum cleaner called Roombas, and InTouch Health designed a new patient friendly robot that wonders into patient hospital rooms and hel ps to remotely diagnosis problems with the help of a physician specialist who may be thousands of miles away. Instead of the patient having to leave the office with a temporary crown, a robot technician is wheeled into the clinic and commands a Cerec machine which takes a digital impression. The Cerec trained dentist then designs the dental crown on a computer screen and wirel essly emails the design to a cad-cam, dental robot located in another room. In about 15 minutes of fascinating buzzing and whirring a perfectly shaped and colored dental crown or onlay is almost magically produced and can be bonded to the tooth in one visit. I nvisalign orthodontics is yet another example of robotic type, dental treatment available from your hi tech

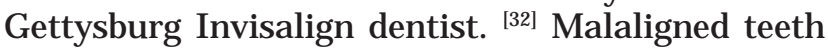
are digitally recreated and then digitally straightened, allowing a series of tooth straightening dental aligners to be robotically fabricated.

\section{Robotic Chewing Simulator for Dental Materials Testing}

The robot was built in collaboration with Società Graal Tech and was financed by Ministry of University Instruction and Research, I taly under the Research of National I nterest Project. It is based on a three-dimensional mechanism with six linear actuators that have been programmed using clinically obtained data to replicate the motion and forces sustained by teeth within a human mouth. This means it replicates natural movement with six degrees of freedom, translating and rotating along each of the Cartesian axes. It is composed of 2 distinct systems: The first is the system driving and controlling the robot, al ong with the robot itself; and the second system collects the data. The controller system is an industrial computer that gives orders to the movable part of the robot, i.e., the Stewart platform, and controls the movements executed, thanks to feedback signals. The Stewart platform is a parallel mechanism made up of a rigid upper body, or moving platform (the end effector simulating the mandible), which is connected to a fixed base by 6 identical kinetic legs, which are equidistant to each other and symmetrically arranged so as to form 2 equilateral triangles on a fixed base. The new robot has the potential to dramatically improve the process of development and testing of new dental materials.

\section{Conclusion}

Robotics could offer dentistry improved accuracy, predictability, safety, quality of care and 
speed of treatment. It has the potential to alter people's quality of dental health in just few years. One might wonder why robots have not yet been introduced to dentistry, as the functions needed are relatively simple. An explanation could be that robotics in dentistry is an example of a disruptive technology, meaning that the current manufacturers of dental equipment might fear a negative effect on their current business and thealienation of dentists, as robots might be seen as a threat to dental professionals. With the emergence of new technologies, future of dentistry is unpredictable. Main concern lies in the vision and feasibility of adapting these technologies in day today teaching and clinical practice.

Any new therapeutic innovation is critical to our future health and such an innovation will, at least initially, cost more than the previous therapy. To abandon the search for improved therapies on the basis of cost would represent enormous disservice to our patients and would distinguish attempts to improve patient care from the quest for better automobiles, audio systems, or computers, or from any area of human endeavor.

\section{Reference}

1. http://oxforddictionaries.com/definition/english/ robotics?q=robotics

2. Palep J H (2009) Robotic assisted minimally invasive surgery. J Min Access Surgery 5(1):1-7.

3. http://www.robots.com/education

4. Maass H, Chantier BB, Cakmak HK. F undamentals of force feedback and application to a surgery simulator. Comput Aided Surg 2003; 8:283-291.

5. Kwoh YS, Hou J, J onckheere EA, Hayall S. A robot with improved absolute positioning accuracy for CT guided stereotactic brain surgery. IEEE Trans. Biomed. Engng 1998; 35(2):153-161.

6. McConnell PI, Schneeberger EW, Michler RE. History and devel opment of robotic cardiac surgery. Problems in General Surgery 2003; 20(2):20-30.

7. Leslie Versweyveld. ZEUS robot system reverses sterilization to enable birth of baby boy. Virtual Medical Worlds Monthly Sep 29, 1999.

8. http://www.ucalgary.ca/news/may2008/neuroArm http:// en.wikipedia.org/wiki/Robotic_surgery

9. http://www.sgistudy.com/Robotic.htm

10. www.diginfo.tv/v/11-0139-r-en.php

11. http://geminoid.dk/

12. http://gizmodo.com/5862512/simroid-trains-future-humanslaves-in-robot-dental-hygiene

13. http://www.diginfo.tv/v/11-0245-r-en.php

14. Gutmann J L, Lovdahl PE. Problems Encountered in Tooth I solation and Access to the Pulp Chamber Space. In Gutmann J L, Dumsha TC, Lovdahl PE, H ovland EJ , editors: Problem Solving in Endodontics-Prevention, I dentification, and Management, 3 edn, St Louis, Missouri, The C.V. Mosby, 1997, pg 47-67.

15. Burns RC, Herbranson EJ. Tooth Morphology and Cavity Preparation. In Cohen S Burns, RC, Editors: Pathways of the Pulp, 7edn, St Louis, Missouri, The C. V. Mosby, 1997 pg 150-202.

16. West J D, Roane J B. Cleaning and Shaping the Root Canal System. In Cohen S Burns, RC, Editors: Pathways of the Pulp, e7 edn, St Louis, Missouri, The C.V. Mosby, 1997, pg 203-257.

17. Dong J . Rule-based Planning for Automated Endodontic Treatment - From Dental Radiography, 3-D Computer Modeling, to Tool Selection and Path Control. Dissertation, Columbia University 2003; 149-153.

18. Schulz MJ, Shao VN, Yun Y. Nanomedicine Design of Particles, Sensors, Motors, Implants, Robots, and Devices, Artech House, 2009; 10.

19. Freitas J r RA. Nanodentistry. J Am Dent Assoc 2000; 131:1559-1566.

20. Mjor I.A, Nordahl I. The density and branching of dentinal tubules in human teeth. Arch Oral Biol 1996; 41(5):401-412.

21. Sumikawa DA, Marshall GW, Gee L. Microstructure of primary tooth dentin Paediatric Dentistry 1999; 21(7):439 $-444$.

22. Speich J E, Rosen J. Medical Robotics. Encyclopedia of Biomaterials and Biomedical Engineering 2004; 983-993.

23. DiGioia AM, Colgan BD, Koerbel N. Computer aided surgery. In: Satava RM, editor. Cybersurgery: Advanced Technologies for Surgical Practice. New York: J ohn Wiley \& Sons; 1998; 121-139.

24. Lueth TC, Hein A, Albrecht J, Demirtas $M$, Zachow $S$, Heissler E, Klein M, Menneking H, Hommel G, Bier J . A Surgical Robot System for Maxillofacial Surgery. IEEE Int. Conf. on I ndustrial Electronics, Control, and Instrumentation (IECON), Aachen, Germany, 1998; 2470-2475.

25. H. Dr. Ing WS, “3D Planning System for Dental Implantology.

26. Xiaojun WC, Yanping L. A Computer-Aided Oral I mplantology System. IEEE Engineering in Medicine and Biology 27th Annual Conference, Shanghai, China, 2005; 3312-3315.

27. Zheng G, Li X, Zhang J, Gu L. Computer-assisted Preoperative Planning and Surgical Navigation System in Dental I mplantology. Proc. of 6th International Special Topic Conference on Information Technology Applications in Biomedicine (ITAB'07), Tokyo, J apan 2007; 139-142.

28. Shapira L. I mage Guided I mplantology - Real-time Guidance of Dental I mplant Surgery in the Operative Field using CTscan image. Computer Assisted Radiology and Surgery 2002; 6: 959-964.

29. Hoffmann WJ, Roman GG, Reinert S. Accuracy of navigation-guided socket drilling before implant installation compared to the conventional free-hand method in a synthetic edentulous lower jaw model. Clinical Oral I mplantology 2005; 16:609-14.

30. Edinger $\mathrm{DH}$. Planning and implant positioning by a robot system in the dental practice. Digital Dental News 2012; 6:32-38.

31. Tal H, Schicho KA, Shohat M. Implant Locating and Placement Based on a Novel Tactile Imaging and Registration Concept: A Technical Note. Int J Oral Max I mplant 2007; 22(6):1007-1011.

32. http://www.robotxworld.com/topics/robotics/articles/130220cerec-ac-robot-introduced-china-shesays.htm 